\begin{tabular}{|c|c|}
\hline Title & Beam scan using the quasi-optical antenna mixer array \\
\hline Author(s) & Nishimura, Toshihiko; Ishii, Nozomu; Itoh, Kiyohiko \\
\hline Citation & $\begin{array}{l}\text { IEEE TRANSA CTIONS ON ANTENNA S A ND PROPA GA TION, 47(7), 1160-1166 } \\
\text { https://doi.org/10.1109/8.785747 }\end{array}$ \\
\hline Issue Date & $1999-07$ \\
\hline Doc URL & http:/hdl .handle.net/2115/5588 \\
\hline Rights & $\begin{array}{l}\text { @1999 IEEE. Personal use of this material is permitted. However, permission to reprint/republish this material for } \\
\text { advertising or promotional purposes or for creating new collective works for resale or redistribution to servers or lists, } \\
\text { or to reuse any copyrighted component of this work in other works must be obtained from the IEEE." }\end{array}$ \\
\hline Type & article \\
\hline File Information & ITAP47-7.pdf \\
\hline
\end{tabular}

Instructions for use 


\section{Beam Scan Using the Quasi-Optical Antenna Mixer Array}

Toshihiko Nishimura, Member, IEEE, Nozomu Ishii, Member, IEEE, and Kiyohiko Itoh, Senior Member, IEEE

\begin{abstract}
The quasi-optical antenna mixer system transforms an RF signal into an intermediate frequency signal just after it spatially receives the $R F$ signal and the local oscillator's (LO) signal. In this paper, we present a novel beam-scanning system using the quasi-optical antenna mixer array and describe its principle of operation and the experimental results in the microwave band. The arrival direction of RF signals can be controlled by adjusting the $L O$ incident angle and/or the $L O$ frequency without implementing RF circuits such as phase shifters. Therefore, the novel beam-scanning system has no loss due to RF circuits and can be easily designed because of its simple composition.
\end{abstract}

Index Terms-Beam scanning, coplanar waveguide, microstrip antenna, phased array, quasi-optical antenna mixer.

\section{INTRODUCTION}

$\mathbf{I}$ $\mathrm{N}$ beam scanning, a phased-array system [1], [2] such as that shown in Fig. 1(a) has generally been used. In this system, some antenna elements are arranged so that phase shifters electrically switch their phases at RF. The conduction loss of the phase shifter increases as frequency increases, especially in the millimeter-wave band. If digital phase shifters are used, quantized phase errors occur. In this paper, we present a novel beam-scanning technique using the quasi-optical antenna mixer [3]-[6] array, as shown in Fig. 1(b), which overcomes the above problems. In Fig. 1, the components fringed by a thick line, thin line, and double line indicate are those designed for RF, local oscillator's frequency (LO) and intermediate frequency (IF), respectively. Therefore, phase shifters can be removed and the feeding circuit can be designed for a lower frequency if this quasi-optical antenna mixer array is used.

Transmission lines between the antennas and mixer are necessary for the conventional receiving system and they cause more loss as the frequency increases. This is a serious problem in low-loss design in the millimeter-wave band. The transmission line should ideally be removed from the system. We propose the use of a quasi-optical antenna mixer, which consists of antennas and a mixer to realize a receiving system that has no transmission line. The quasi-optical antenna mixer transforms the RF signal, which is spatially received with the

Manuscript received June 8, 1998; revised February 8, 1999. This work was supported by a Grant-in-Aid for Scientific Research (A) (2) 07405019 from the Ministry of Education, Science, and Culture of Japan.

T. Nishimura and K. Itoh are with the Graduate School of Engineering, Hokkaido University, Sapporo, 060-8628 Japan.

N. Ishii is with the Biocybernetics Engineering Department, Niigata University, Niigata, 950-2181 Japan.

Publisher Item Identifier S 0018-926X(99)07070-2.

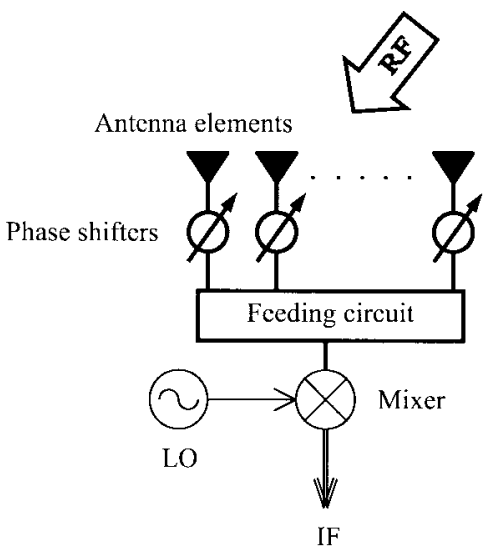

(a)

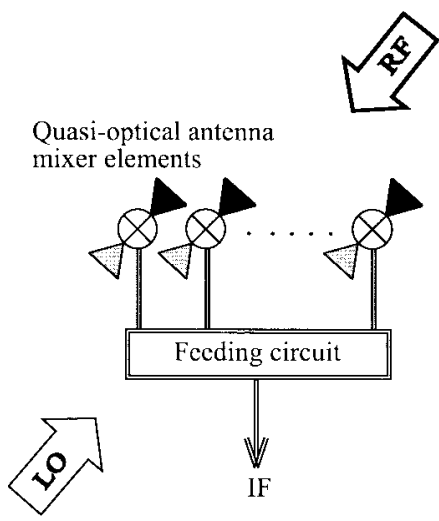

(b)

Fig. 1. Schematic of beam-scanning system. (a) A conventional phased-array system. (b) A quasi-optical antenna mixer array.

LO into an IF signal. No transmission loss can occur because there is no transmission line.

In this paper, we describe the principle of the novel beamscanning technique and show the validity of this new technique through experimental results. First, the principle of the beam scanning is theoretically derived, and the scanning direction can be controlled by adjusting the LO incident angle and/or LO frequency. Second, a new microstrip antenna that is fed by a coplanar waveguide and has two patches on both sides of the grounded substrates, is designed. This antenna can separately receive different frequencies and it is used in our novel quasi-optical antenna mixer. Finally, a four-element quasi-optical antenna mixer is fabricated and experimented in microwave band to check the validity of our novel beamscanning technique. 


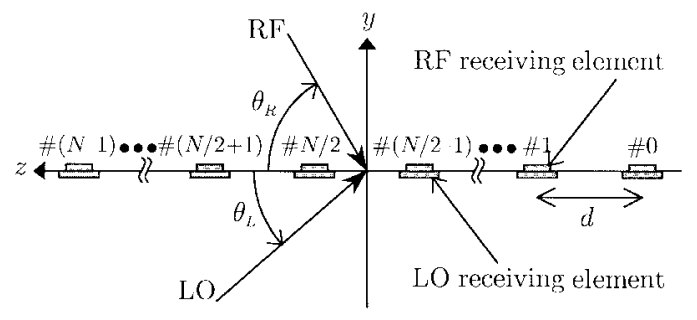

Fig. 2. Arrangement of elements and coordinate.

\section{PRINCIPLE OF THE BEAM SCANNING}

\section{A. Formulation}

Fig. 2 shows the arrangement of our novel quasi-optical antenna mixer array. The elements are uniformly arranged along the $z$ axis. The number of elements is denoted by $N$. The origin is defined as the center of the array.

To simplify our discussion, we only consider the $y z$ plane, although it can be expanded to three dimensions and we assume that influences in a field viewpoint, e.g., mutual coupling between the elements, are ignored. The RF and LO waves are incident in the upper half-space $(y>0)$ and the lower half-space $(y<0)$, respectively. As shown in Fig. 2, the incident angles $\theta_{R}$ and $\theta_{L}$ are defined as the angles formed between the positive direction of the $z$ axis and the RF and LO arrival directions, respectively. The RF amplitude on the $n$th element $E_{R, n}$ is expressed as

$$
E_{R, n}=S_{R, n} \cdot D_{R, n}
$$

where $S_{R, n}$ is the electric field intensity of the signal arriving at the $n$th element and $D_{R, n}$ is the element pattern of the $n$th element. Similarly, the LO amplitude on the $n$th element $E_{L, n}$ is expressed as

$$
E_{L, n}=S_{L, n} \cdot D_{L, n}
$$

where $S_{L, n}$ is the electric field intensity of the signal arriving at the $n$th element, and $D_{L, n}$ is the element pattern of the $n$th element.

Phase differences between two successive elements for the RF and LO signals, $\varphi_{R}$ and $\varphi_{L}$, respectively, are defined as follows:

$$
\begin{gathered}
\varphi_{R}=k_{R} d \cos \theta_{R} \\
\varphi_{L}=k_{L} d \cos \theta_{L}
\end{gathered}
$$

where $k_{R}$ and $k_{L}$ are wavenumbers for the RF and LO waves, respectively, and $d$ is the element separation between two successive elements and equals a half wavelength of the RF in free-space. Now, a quasi-optical antenna mixer element transforms the RF signal into an IF signal. If an element at the most negative position along the $z$ axis (\#0) is defined as a reference of the phase in the system, the IF output in the $n$th element $I_{n}$ is derived as

$$
\begin{aligned}
I_{n}= & E_{R, n} \cos \left(\omega_{R} t-n \varphi_{R}\right) \otimes E_{L, n} \cos \left(\omega_{L} t-n \varphi_{L}\right) \\
=\frac{1}{2} & K_{n} E_{R, n} E_{L, n} \\
& \times\left[\cos \left\{\left(\omega_{R}-\omega_{L}\right) t-n\left(\varphi_{R}-\varphi_{L}\right)+\beta_{n-}\right\}\right. \\
& \left.\quad+\cos \left\{\left(\omega_{R}+\omega_{L}\right) t-n\left(\varphi_{R}+\varphi_{L}\right)+\beta_{n+}\right\}\right] \\
= & A_{n}\left[\cos \left(\Omega_{-} t-n \Psi_{-}+\beta_{n-}\right)\right. \\
& \left.\quad+\cos \left(\Omega_{+} t-n \Psi_{+}+\beta_{n+}\right)\right]
\end{aligned}
$$

where $\omega_{R}, \omega_{L}$ are angular frequencies of the RF and LO signals, respectively; $K_{n}$ is the conversion gain of the mixer; and

$$
\begin{aligned}
& A_{n}=\frac{1}{2} K_{n} E_{R, n} E_{L, n} \\
& \Omega_{-}=\omega_{R}-\omega_{L} \\
& \Omega_{+}=\omega_{R}+\omega_{L} \\
& \Psi_{-}=\varphi_{R}-\varphi_{L} \\
& \Psi_{+}=\varphi_{R}+\varphi_{L} .
\end{aligned}
$$

The phase fluctuation after mixing, for example, due to a slight difference in the length of the transmission line, is denoted by $\beta_{n-}$ and $\beta_{n+}$. In addition, the symbol $\otimes$ represents the mixing operation, which is defined as the operator to multiply the product of both sides of the symbol by the conversion gain.

These IF outputs are combined by the power combiner. In an ideal Wilkinson power combiner [7], the input signal would be added to the output signals, keeping isolation between the output ports. Therefore, the combined signal $I_{\mathrm{SUM}}$ is obtained as follows:

$$
I_{\mathrm{SUM}}=c \sum_{n=0}^{N-1} I_{n}
$$

where the factor " $c$ " is a constant that depends on the number of elements.

To simplify the discussion, we assume that

- the arrival signal's amplitude and the conversion gain of each mixer are uniform:

$$
A_{n}=A \quad \text { for } n=0,1, \cdots, N-1
$$

- IF outputs have no phase fluctuation after mixing:

$$
\beta_{n-}=\beta_{n+}=0 \quad \text { for } n=0,1, \cdots, N-1 .
$$

Furthermore, by moving the reference of the phase to the physical center of the array, (11) can be

$$
I_{\mathrm{SUM}}=F_{d c} \cos \left(\Omega_{-} t\right)+F_{u c} \cos \left(\Omega_{+} t\right) .
$$

Therefore, $I_{\text {SUM }}$ can be separated into two components: the up and down conversions. These amplitudes are given by

$$
\begin{aligned}
F_{d c}=c A \frac{\sin \left(N \frac{\Psi_{-}}{2}\right)}{\sin \frac{\Psi_{-}}{2}} \\
F_{u c}=c A \frac{\sin \left(N \frac{\Psi_{+}}{2}\right)}{\sin \frac{\Psi_{+}}{2}} .
\end{aligned}
$$






Fig. 3. Relation of $\theta_{R}$ and $\theta_{L}$ as a function of the frequency ratio.

The point is that the IF output amplitudes $F_{d c}$ and $F_{u c}$ behave as a uniform spacing linear array designed at the corresponding frequencies $\Omega_{-}$and $\Omega_{+}$, respectively.

Equations (13) and (14) imply that the amplitude of the IF output is a function of the RF and LO incident angles and the RF and LO frequencies $\left(\theta_{R}, \theta_{L}, f_{R}\right.$, and $\left.f_{L}\right)$, because $f_{R}$ and $f_{L}$ are contained in $\Psi_{-}$and $\Psi_{+}$. This fact suggests that the receiving direction of maximum RF can be controlled by adjusting the parameters $\theta_{R}, \theta_{L}, f_{R}$, and $f_{L}$.

To explain this suggestion more clearly, we begin by examining the case where the element pattern is isotropic and then the case that the typical pattern of the half-resonant microstrip antenna is adapted as the element pattern.

\section{B. Isotropic Element Pattern}

Here it is assumed that the element pattern is isotropic; that is, $A$ is independent of $\theta_{R}$ and $\theta_{L}$ in (13) and (14). The maximum value of $F_{d c}$ in (13) can be derived from the condition that $\Psi_{-}=0$. Therefore, the RF incident angle to maximize $F_{d c},\left.\theta_{R, d c}\right|_{\max }$ is obtained as

$$
\left.\theta_{R, d c}\right|_{\max }=\cos ^{-1}\left(\frac{f_{L}}{f_{R}} \cos \theta_{L}\right) .
$$

Similarly, the maximum value of $F_{u c}$ in (14) can be derived from the condition that $\Psi_{+}=0$. In this case, $\theta_{R}$,uc $\left.\right|_{\max }$ is obtained as

$$
\left.\theta_{R, u c}\right|_{\max }=\cos ^{-1}\left(-\frac{f_{L}}{f_{R}} \cos \theta_{L}\right) \text {. }
$$

Fig. 3 is a plot of (15) and (16) as a parameter of the frequency ratio $f_{L} / f_{R}$. The vertical axes on the left and right correspond to $\left.\theta_{R, d c}\right|_{\max }$ and $\left.\theta_{R, u c}\right|_{\max }$, respectively. Fig. 3 shows that $\left.\theta_{R, d c}\right|_{\max }$ and $\theta_{R},\left.u c\right|_{\max }$ can be uniquely determined for any value of $\theta_{L}$ if $f_{L} / f_{R}$ is fixed. If $\theta_{L}$ is fixed, $\left.\theta_{R, d c}\right|_{\max }$ and $\left.\theta_{R, u c}\right|_{\max }$ can be uniquely determined according to $f_{L} / f_{R}$, except when $\theta_{L}=90^{\circ}$. Typical values of $\left.\theta_{R, d c}\right|_{\max }$ and $\left.\theta_{R, u c}\right|_{\max }$ are listed in the left-hand column of Table I. Thus, the direction of the beam can be controlled by changing the LO incident angle $\theta_{L}$ and/or the frequency ratio $f_{L} / f_{R}$. In practice, $f_{L}$ is changed but $f_{R}$ is not.
TABLE I

\begin{tabular}{|c|c|c|c|c|}
\hline \multirow[b]{3}{*}{$\theta_{L}$} & \multicolumn{2}{|c|}{ isotropic pattern } & \multicolumn{2}{|c|}{ patch pattern } \\
\hline & (15) & (16) & (13) & (14) \\
\hline & $\left.\theta_{R, d r}\right|_{\max }$ & $\left.\theta_{R, u c}\right|_{\max }$ & $\left.\theta_{R, d c}\right|_{m}$ & $\theta_{R, u c}$ \\
\hline $10^{\circ}$ & $28^{\circ}$ & $152^{\circ}$ & $41^{\circ}$ & $139^{\circ}$ \\
\hline $50^{\circ}$ & $55^{\circ}$ & $125^{\circ}$ & $59^{\circ}$ & $121^{\circ}$ \\
\hline $90^{\circ}$ & $90^{\circ}$ & $90^{\circ}$ & $90^{\circ}$ & $90^{\circ}$ \\
\hline
\end{tabular}

$\left.\theta_{R, d c}\right|_{\max }$ AND $\left.\theta_{R, u c}\right|_{\max }$ From (11)-(14)



Down-conversion

(a)

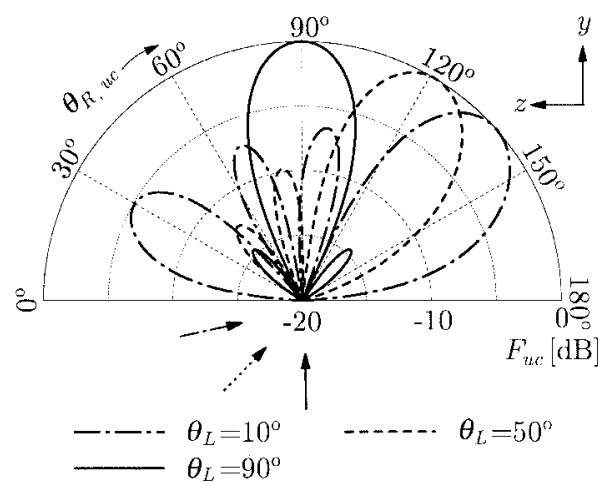

Up-conversion

(b)

Fig. 4. IF output pattern $\left(f_{L} / f_{R}=0.9\right)$. (a) Down conversion. (b) Up conversion.

\section{Patch-Element Pattern}

In (13) and (14), the RF and LO element patterns of the $n$th element, $D_{R, n}$ and $D_{L, n}$, are assumed to be identical, i.e., $D_{R, n}=D_{R}$ and $D_{L, n}=D_{L}$ for $n=0,1, \cdots, N-1$. The model used for calculations is composed of a four-element quasi-optical antenna mixer system $(N=4)$ that has square patches as receiving antennas. The elements are assumed to be linearly arranged with their $H$-plane. We use the typical pattern of a half-resonant squared patch as the element pattern $D_{R}, D_{L}$ [8]. The combined IF outputs are calculated as a function of $\theta_{R}$ when the frequency ratio and the LO incident angle are fixed. In the following, $\theta_{R, d c}$ and $\theta_{R, u c}$ are defined as the RF incident angles corresponding to the components of down and up conversion, respectively. In the following figures, $F_{d c}$ and $F_{u c}$ are normalized by their own maximum value.

1) The Case Where the Frequency Ratio is Fixed: The frequency ratio is fixed at $f_{L} / f_{R}=0.9$. Equations (13) and (14), which include the effect of the element pattern, are calculated when the LO incident angle is set at $10^{\circ}, 50^{\circ}$, and $90^{\circ}$. Fig. 4(a) shows the IF output pattern of the down 


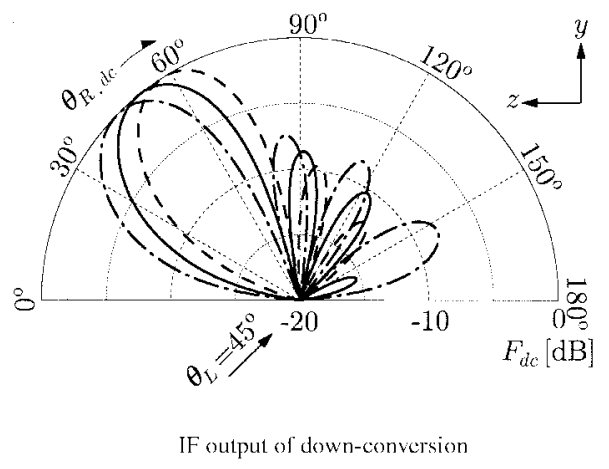

(a)

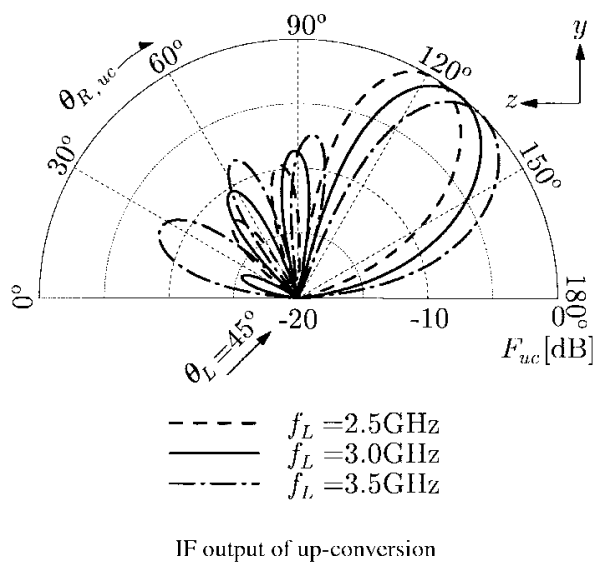

(b)

Fig. 5. IF output pattern $\left(\theta_{L}=45^{\circ}\right)$. (a) IF output of down conversion. (b) IF output of up conversion.

conversion as a function of the RF incident angle and Fig. 4(b) shows its counterpart of up conversion. The IF output pattern is the IF output as a function of the RF incident angle. As the LO incident angle $\theta_{L}$ changes, the RF incident angle to maximize the conversion results also changes. This implies the possibility of beam scanning by $\theta_{L} .\left.\theta_{R, d c}\right|_{\max }$ and $\left.\theta_{R, u c}\right|_{\max }$ in Fig. 4(a) and (b) are listed in the right-hand column of Table I. It is obvious that changing the LO incident angle enables the RF incident angle to be scanned.

From Fig. 4 and Table I, it can be seen that the IF outputs including the element pattern become smaller as the LO incident angle approaches the $z$-axis. The reason why $\left.\theta_{R, d c}\right|_{\max }$ and $\left.\theta_{R, u c}\right|_{\max }$ shift to the $y$-axis $\left(\theta=90^{\circ}\right)$ when $\theta_{L}$ is close to $0^{\circ}$ or $180^{\circ}$ is due to the $H$-plane pattern of the patch.

2) The Case Where the LO Incident Angle is Fixed: Similarly, changing the LO frequency also enables $\left.\theta_{R, d c}\right|_{\max }$ and $\theta_{R},\left.u c\right|_{\max }$ to be scanned when the LO incident angle is fixed, except when $\theta_{L}=90^{\circ}$. Fig. 5 shows the IF output pattern at $\theta_{L}=45^{\circ}$ as the LO frequency is changed. For LO frequency ranging from 2.5 to $3.5 \mathrm{GHz},\left.\theta_{R, d c}\right|_{\max }$ is scanned from 58 to $46^{\circ}$, and $\left.\theta_{R, u c}\right|_{\max }$ is scanned from 122 to $134^{\circ}$. Both ranges of scanned angle are $12^{\circ}$.

These above results lead to the conclusion that our novel system can control the RF arrival direction by changing the LO incident angle and/or the LO frequency without highfrequency circuits such as phase shifter.

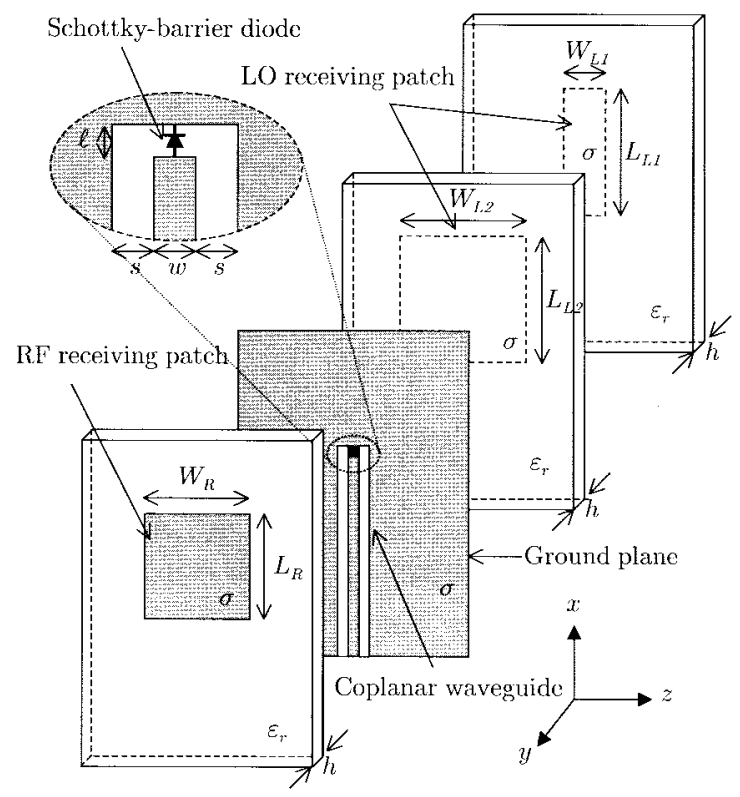

Fig. 6. Configuration of quasi-optical antenna mixer element.

\section{EXPERIMENT}

The results presented in the previous section are experimentally verified in this section. First, a quasi-optical antenna mixer element, which has a novel construction, is described. Second, our experimental environment is explained. The experiment is performed in the microwave band. Finally, the experimental results of scanning are presented.

\section{A. Quasi-Optical Antenna Mixer Element}

To realize a quasi-optical antenna mixer such as that shown in Fig. 1(b), the RF and LO signals must be received near the mixer. A wide-band antenna that can receive both RF and LO signals has generally been used in a quasi-optical antenna mixer. In this case, there are spatial limitations on the system design because the RF and LO waves must be incident from one side of the substrate. It is possible, for example, that irradiation of the LO wave interferes with the RF incident wave.

Therefore, we propose a novel design to overcome the above limitations. Fig. 6 shows the configuration of our quasi-optical antenna mixer element, and the parameters of the model are listed in Table II. The RF receiving patch is operated at the frequency of $f_{R}=3.0 \mathrm{GHz}$. On the other face, the LO receiving patches are stacked to operate at two frequencies: $f_{L 1}=2.7 \mathrm{GHz}$ and $f_{L 2}=3.3 \mathrm{GHz}$. Therefore, the frequency of down conversion is $0.3 \mathrm{GHz}$ because $\mathrm{RF}$ is $3.0 \mathrm{GHz}$. The reason why the LO frequencies are determined above is that the IF circuit does not need to be designed for a wide band. If a wide-band design is adopted for an LO receiving patch, sweeping the LO frequency would, in principle, enable continuous scanning of the beam.

The coplanar waveguide on a ground plane between dielectric substrates is designed to be $50 \Omega$ and the end is opened as shown in Fig. 6. Three patches are "offset" along the $x$-axis. The value of "offset" is defined as the distance between the end 
TABLE II

Parameters of the ExPerimental Model

\begin{tabular}{lc|rl}
\multicolumn{4}{c}{ Substrates } \\
\hline parmittivity & $\varepsilon_{r}$ & 10.2 & \\
& $\tan \delta$ & 0.0023 & \\
thickness & $h$ & 0.6 & {$[\mathrm{~mm}]$} \\
conductivity & $\sigma$ & $5.8 \times 10^{7}$ & {$[\mathrm{~S} / \mathrm{m}]$} \\
thickness of conductor & $t$ & 17.5 & {$[\mu \mathrm{m}]$} \\
\hline
\end{tabular}

\begin{tabular}{lc|rl}
\multicolumn{4}{c}{$\mathrm{RF}$ patch } \\
\hline frequency & $f_{R}$ & 3.0 & {$[\mathrm{GHz}]$} \\
length & $L_{R}$ & 15.6 & {$[\mathrm{~mm}]$} \\
width & $W_{R}$ & 15.6 & {$[\mathrm{~mm}]$} \\
offset & $x_{R}$ & 5.2 & {$[\mathrm{~mm}]$} \\
\hline
\end{tabular}

\begin{tabular}{lc|rc}
\multicolumn{4}{c}{ LO1 patch } \\
\hline frequency & $f_{L 1}$ & 2.7 & {$[\mathrm{GHz}]$} \\
length & $L_{L 1}$ & 19.0 & {$[\mathrm{~mm}]$} \\
width & $W_{L 1}$ & 5.0 & {$[\mathrm{~mm}]$} \\
offset & $x_{L 1}$ & 5.0 & {$[\mathrm{~mm}]$} \\
\hline
\end{tabular}

\begin{tabular}{lc|rl}
\multicolumn{4}{c}{ LO2 patch } \\
\hline frequency & $f_{L 2}$ & 3.3 & {$[\mathrm{GHz}]$} \\
length & $L_{L 2}$ & 14.2 & {$[\mathrm{~mm}]$} \\
width & $W_{L 2}$ & 14.2 & {$[\mathrm{~mm}]$} \\
offset & $x_{L .2}$ & 4.4 & {$[\mathrm{~mm}]$} \\
\hline
\end{tabular}

\begin{tabular}{lc|cc}
\multicolumn{4}{c}{ Coplanar waveguide } \\
\hline inner-conductor & $w$ & 0.80 & {$[\mathrm{~mm}]$} \\
slit & $s$ & 0.65 & {$[\mathrm{~mm}]$} \\
gap & $\ell$ & 0.30 & {$[\mathrm{~mm}]$} \\
\hline
\end{tabular}

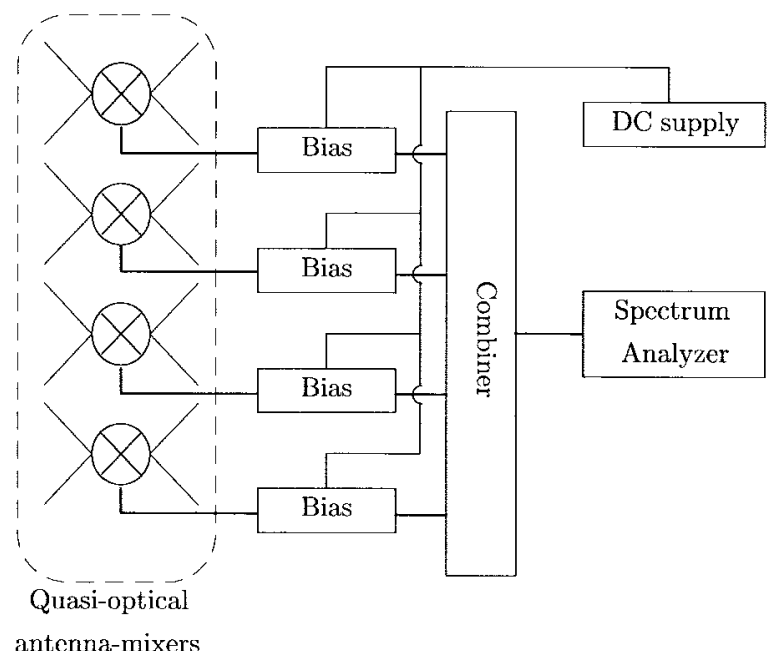

antenna-mixers

Fig. 7. Block diagram of four-element quasi-optical antenna mixer system.

of the coplanar waveguide and the center of the patch along the $x$-axis and these values are listed in Table II. The offset makes the patches and the coplanar waveguide match because each patch is electromagnetically coupled with the open end of the coplanar waveguide.

\section{B. Four-Element Array and Experimental Environment}

Fig. 7 shows a block diagram of a four-element quasioptical antenna mixer system. The elements are linearly arranged along the $z$-axis at 5-cm intervals (a half wavelength of the RF). A Schottky-barrier diode (SANYO Electric Co. Ltd.; SBL-121) is mounted in the gap of the coplanar waveguide's end as a mixer diode, as shown in Fig. 6. The IF outputs

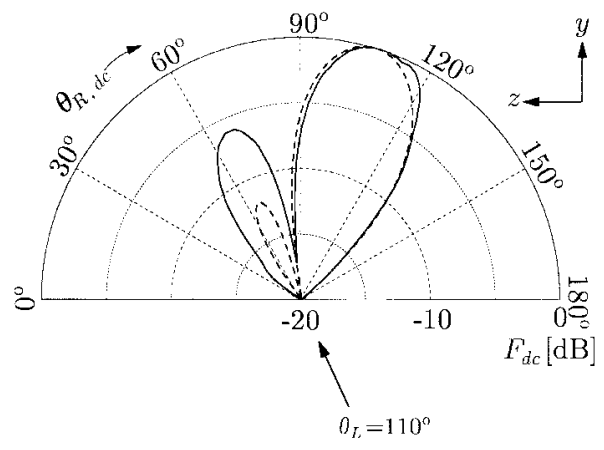

$f_{L l}=2.7 \mathrm{GHz}$

(a)

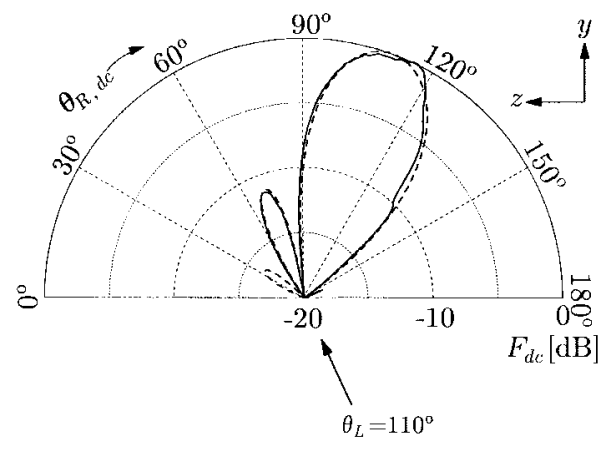

$f_{L 2}=3.3 \mathrm{GHz}$

solid line: meas. broken line: calc.

(b)

Fig. 8. Comparison between measured and calculated IF output patterns. (a) $f_{L 1}=2.7 \mathrm{GHz}$. (b) $f_{L 2}=3.3 \mathrm{GHz}$.

from each diode are stably performed by bias current because the bias block has a constant current circuit using an field effect transistor (FET). In addition, the entire IF outputs are combined by a Wilkinson power combiner. In the experiment, RF, LO, and other outputs are observed with the IF outputs using a spectrum analyzer, because no filter is used.

Measurements are carried out in an anechoic chamber. Two standard horn antennas are placed in front of and at the rear of the antenna mixer array to irradiate the RF and the LO signals in the space. The distances between the array and two horn antennas are $4.65 \mathrm{~m}$ for the RF and $0.3 \mathrm{~m}$ for the $\mathrm{LO}$.

The conversion gain of a single element is about $-10 \mathrm{~dB}$ [9]. Details of the mixing properties and the experimental environment of a single element are reported in [9].

\section{Experimental Results}

In order to obtain the following calculated data, the element pattern is evaluated using an electromagnetic simulator. Although only the down conversion is discussed here, the up conversion is analogous in beam scanning.

Fig. 8 shows a comparison between measured and calculated IF output patterns at $\theta_{L}=110^{\circ}$. Fig. 8(a) and (b) is derived by setting the $\mathrm{LO}$ frequency at $f_{L 1}=2.7 \mathrm{GHz}$ and $f_{L 2}=3.3 \mathrm{GHz}$, respectively. The solid line denotes the measured pattern and the broken line denotes the calculated pattern from (13). The measured pattern agrees with the 


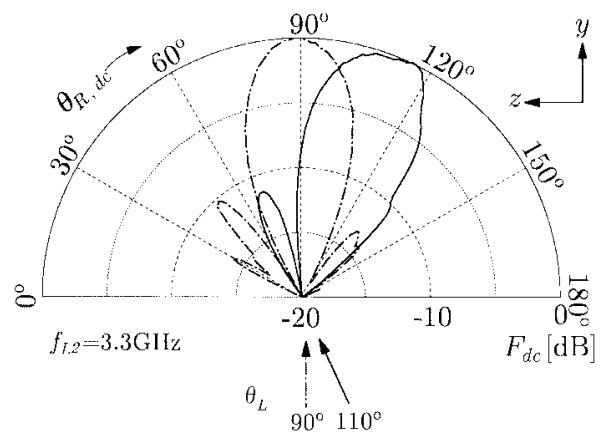

Fig. 9. Beam scanning by changing the LO incident angle through the measurement.

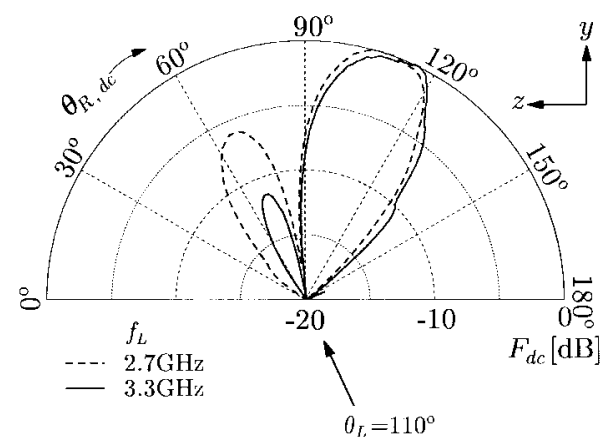

Fig. 10. Beam scanning by changing the LO frequency through the measurement.

calculated pattern in terms of the direction of the mainbeam and the beamwidth, although behavior of each element in combination with its neighbors and finite substrate is ignored. However, there are some errors in the sidelobe level because the characteristics of the elements may not be uniform. The nonuniformity is due to the manufacturing process.

Fig. 9 shows the experimental behavior of the beam scanning by changing the LO incident angle. It compares output patterns of $\theta_{L}=90^{\circ}$ (dot-dashed line) and $\theta_{L}=110^{\circ}$ (solid line) at $f_{L 2}=3.3 \mathrm{GHz}$. In this case, the steering range of $\left.\theta_{R, d c}\right|_{\max }$ is from $90^{\circ}$ to $116^{\circ}$. Moreover, in the case of similar measurement at $f_{L 1}=2.7 \mathrm{GHz}$, the steering range of $\left.\theta_{R, d c}\right|_{\max }$ is from $90^{\circ}$ to $108^{\circ}$.

Fig. 10 shows the experimental behavior of the beam scanning by changing the LO frequency. It is a comparison of output patterns at $f_{L 1}=2.7 \mathrm{GHz}$ (solid line) and $f_{L 2}=3.3$ $\mathrm{GHz}$ (broken line) when $\theta_{L}=110^{\circ}$. In this case, $\left.\theta_{R, d c}\right|_{\max }$ ranges from 108 to $116^{\circ}$.

Fig. 11 shows $\theta_{L}$ versus $\left.\theta_{R, d c}\right|_{\max }$ for the LO frequencies of $f_{L 1}=2.7 \mathrm{GHz}$ and $f_{L 2}=3.3 \mathrm{GHz}$. In the figure, the solid and broken lines are the calculated values at $f_{L 1}=2.7 \mathrm{GHz}$ and $f_{L 2}=3.3 \mathrm{GHz}$ from (13), respectively. The circular and triangular symbols indicate the measured values at $f_{L 1}$ and $f_{L 2}$, respectively. This figure shows the possibility of beam scanning by changing the LO incident angle and/or the LO frequency.

\section{CONCLUSION}

The results of this study lead us to make the following conclusions about the characteristics of beam scanning of our novel array system.



Fig. 11. The RF incident angle as a function of the LO incident angle and frequency.

- The scan angle varies almost linearly according to the LO frequency when the LO incident angle is fixed, except when $\theta_{L}=90^{\circ}$.

- The scan angle varies almost linearly according to the LO incident direction when the LO frequency is fixed.

- The larger the frequency ratio is or the closer to the $z$ axis the LO incident angle is, the larger the range of the scan angle.

In conclusion, a novel beam-scanning technique is proposed by using the quasi-optical antenna mixer array and its principle of operation is explained. To realize the technique, a new quasi-optical antenna mixer, in which coplanar waveguide feed and RF/LO separating inputs are on both sides of the substrate, was developed. In addition, a four-element quasioptical antenna mixer array was constructed, and tested in the microwave band. The experimental results agree with the theoretical ones, thus verifying that the arrival direction of the RF signal can be controlled by this array without RF circuits such as phase shifters.

\section{REFERENCES}

[1] R. Tang and R. W. Burns, "Phased array," in Antenna Engineering Handbook, 3rd ed., R. C. Johnson, Ed. New York: McGraw-Hill, 1993, ch. 20.

[2] W. D. Wirth, "Signal processing for target detection in experimental phased array radar ELRA," Proc. Inst. Elect. Eng., vol. 128, pt. F, pp. 311-316, 1981.

[3] C. Y. Lee and T. Itoh, "Active integrated antenna and quasi-optical technique: Fundamentals and applications," in Asia Pacific Microwave Conf., Tutorial Session 03, Oct. 1995, pp. 89-189.

[4] B. K. Kormanyos, P. H. Ostdiek, W. L. Bishop, T. W. Crowe, and G. M. Rebeiz, "A planar wide-band 80-200 GHz subharmonic receiver," IEEE Trans. Microwave Theory Tech.—Special Issue Quasi-Opt. Tech., vol. 41, pp. 1661-1863, Oct. 1993.

[5] W. Chujo, H. Shimakage, and B. Komiyama, "A high-Tc superconducting active slot antenna with a YBCO step-edge Josephson junction array," in Asia Pacific Microwave Conf., Dec. 1994, pp. 1081-1084.

[6] K. Yamaguchi, K. Hayashi, K. Itoh, K. Suzuki, and Y. Enomoto, "Analysis of a millimeter-wave microstrip antenna for a Josephson mixer," Tech. Rep. IEICE, SCE95-7, MW95-7, pp. 37-42, Apr. 1995.

[7] E. Wilkinson, "An N-way hybrid power divider," IRE Trans. Microwave Theory Tech., vol. MTT-8, pp. 116-118, Jan. 1960.

[8] C. A. Balanis, Antenna Theory-Analysis and Design. New York: Wiley, 1997, ch. 14, pp. 722-784.

[9] T. Nishimura, N. Ishii, and K. Itoh, "Dual frequency operation microstrip antenna system fed by coplanar-waveguide," Advances in Superconductivity, vol. IX/2, pp. 1265-1268, 1996. 




Toshihiko Nishimura (M'98) received the B.S. and M.S. degrees in physics and the Ph.D. degree in electronics engineering all from Hokkaido University, Sapporo, Japan, in 1992, 1994, and 1997, respectively.

In 1998, he joined the Graduate School of Engineering at Hokkaido University, where he is currently a Research Associate of the Electronics and Information Engineering Division. His current research interests are in the millimeter-wave antenna system.

Dr. Nishimura is a member of the IEICE of Japan and the Physical Society of Japan.



Nozomu Ishii (S'90-M'92) was born in Sapporo, Japan, on October 8, 1966. He received the B.S., M.S., and Ph.D. degrees from Hokkaido University, Sapporo, Japan, in 1989, 1991, and 1996, respectively.

In 1991, he joined the faculty of Engineering at Hokkaido University. Since 1998, he has been with the faculty of Engineering at Niigata University, Japan, where he is currently an Associate Professor of the Biocybernetics Engineering Department. His current research interests are in the area of small antenna and millimeter-wave antenna.

Dr. Ishii is a member of the IEICE of Japan.



Kiyohiko Itoh (M'71-SM'96) was born in Sapporo, Japan, on May 15, 1939. He received the B.S.E.E., M.S., and Ph.D. degrees from Hokkaido University, Sapporo, Japan, in 1963, 1965, and 1973, respectively.

Since 1965, he has been a Faculty Member of Engineering, Hokkaido University, where he is currently a Professor of the Electronics and Information Engineering Division. He has been a member of the Senate of Hokkaido University since June 1997. From 1970 to 1971 he was with the Department of Electrical and Computer Engineering, Syracuse University, Syracuse, NY, as a Research Associate on leave from Hokkaido University. His special interests are in electromagnetic radiation, wave optics, mobile radio communications, and solar power satellites.

Dr. Itoh is a member of the IEICE of Japan and the Institute of Image Information and Television Engineers of Japan. He was a Chairman of the 1996 International Symposium on Antennas and Propagation (ISAP'96). He received the Solar Power Satellite (SPS) Award in 1991. 